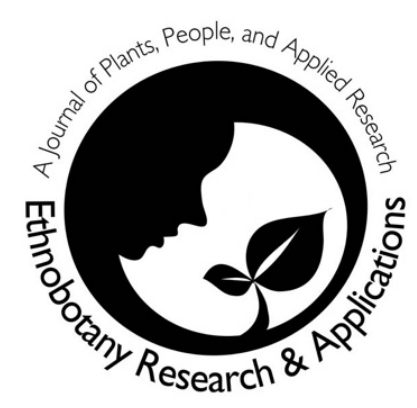

\title{
Ethnobotany of the Mountain Regions of Far Eastern Europe I Ethnobotany of the Mountain Regions of Central Asia and Altai - a review
}

\section{Book review}

Ethnobotany of the Mountain Regions of Far Eastern Europe-Ural, Northern Caucasus, Turkey, and Iran. Ketevan Batsatsashvili, Zaal Kikvidze, Rainer W. Bussmann (Eds). Springer Nature Switzerland AG 2020. xxix + 1063 pp. Price: $€$ 415.99, ISBN 978-3-030-28939-3.

Ethnobotany of the Mountain Regions of Central Asia and Altai. Ketevan Batsatsashvili, Zaal Kikvidze, Rainer W. Bussmann (Eds). Springer Nature Switzerland AG 2020. xxii + 881 pp. Price: $€$ 291.19, ISBN 978-3-030-28946-1.

Have you ever imagined how many efforts are required to keep a cultural identity alive? Traditional knowledge, for example, has no doubt gained renewed importance these days, but to make such knowledge available to a wider audience, extensive efforts are required. The books under review are the result of such efforts from their editors who are eminent and renowned ethnobotanists of world.

Both the above books are written on the same subject (ethnobotany of the mountain regions), edited by the same editors (Ketevan Batsatsashvili, Zaal Kikvidze and Rainer W. Bussmann), with same presentation style, published by the same publisher (Springer International Publishing) in the same year (2020). So there is a lot of similarity between these books and it will be better to review them together side by side.

As already mentioned, these books are a part of book series Ethnobotany of Mountain Regions
(ETMORE,

https://link.springer.com/bookseries/15885). Eight volumes of this book series have been published so far in the current year (2020).

\section{Correspondence}

Zubair A. Malik

Department of Biology, Govt. HSS Harduturoo Anantnag (J\&K) India 192201

*Corresponding Author:

malikmzubair081@gmail.com

\section{Ethnobotany Research \& Applications}

20:36 (2020)

The discipline of ethnobotany has received a lot of attention during the recent past and many individual ethnobotanical studies are now available from many regions of the world. The books under review are very special in the sense that these are comprehensive and encyclopedic that have amassed the vast but scattered ethnobotanical studies of worlds mountain regions. Such books are very rare and are not easily available in the market.

The books aim to present (and document) the comprehensive and thorough introduction to the plant use knowledge of the inhabitants of their respective mountain regions (Far Eastern Europe and Central Asia \& the Altai). The contents of both the books are divided into two parts viz., Part I (Regions) and Part II (Plant profiles). Part I of both 
the books gives a detailed description of surveyed areas/mountain regions regarding their topography, climate, geology and vegetation.

Part II, with many chapters describing the plant profiles, forms the bulk (96-98\%) of these books. While part II of Ethnobotany of Central Asia and Altai contains 148 chapters (describing 258 plant species) contributed by 55 well-known ethnobotanists of different regions (China, Georgia, Norway, Sweden, Tajikistan), Ethnobotany of Far Eastern Europe describes the profiles of 243 plant species under 144 chapters contributed by 12 significant contributors from Georgia, Sweden and Iran. Ethnobotany of more than 440 plant species is documented in these two books (243 species from Far Eastern Europe and 258 from Central Asia and Altai). About 55 plant species are common in both volumes.

Each chapter of Part /I is dedicated either to a particular species of a family (e.g. Achnatherum splendens of Poaceae) or a few species of the same genus of a particular family (e.g. four species of genus Achillea i.e. Achillea asiatica; A. filipendulina; $A$. millefolium and $A$. setacea of Asteraceae). The plant species covered are arranged in an alphabetic order, irrespective of the family to which they belong. The chapters present the detailed and comprehensive information on various aspects of plant species including the current overview of taxonomy, synonyms, local names, botanical description, information on the ecology and distribution, local medicinal, handicraft and other uses. Phytochemistry of most of the described species is also provided. The description of plants is amazing wherein very minute details of morphological characters and major events of the life cycle (flowering\& fruiting) are given. This along with high-quality photographs (taken by the editors and/or contributors) makes the books an ideal and reliable pictorial field guide that would help the students/researchers in identifying and locating these plants. The already documented but scattered information on various uses of plants (medicinal, food, handicrafts and others) is amassed nicely. The ethnobotanical particulars have data from both the past and the most recent scientific studies of plant use in the regions. The literature sources are up to date.

Traditional knowledge has played a key role in the development of new drugs in modern therapeutic system. The editors/contributors could have described another aspect of the plants under the heading 'Drug Discovery' wherein they could have shared the successful and/or unsuccessful attempts of drug discovery from them based on traditional knowledge. This was especially possible for the plants whose phytochemistry is known. Such a section could have highlighted and strengthened the link between traditional knowledge and drug discovery.

The climate of the two mountain regions (Far Eastern Europe and Central Asia \& Altai) is different as described in Part I of both books and since the plant morphological traits are affected by varied climates in different regions of the world, the morphology of plants that are common to both the regions could differ. But no such variation is mentioned anywhere, and the botanical description of common plants is the same. While describing the species that are common to both the regions, the authors could have compared their morphological characters. For example, "plant species $X$ of Far Eastern Europe is taller than/shorter than/leaves are narrow/broader that the same species found in Central Asia \& Altai".

Bunium persicum is one of the species that are common to both books, but in Far Eastern Europe, its family is wrongly shown as Brassicaceae (P 233) while it belongs to Apiaceae as correctly shown in the book of Central Asia and Altai (P 175). Some of the references are wrongly cited as, for example, a work (Bhat et al., 2015) from Western Himalaya (India) is mentioned many times throughout the book should be Malik et al., 2015 and not Bhat et al., 2015. The editors/contributors must have missed these minor mistakes due to oversight.

Despite such small mistakes these are the bestedited books on the subject. These books are too expensive for personal collection, but educational institutes and research organizations can make them available/accessible to the concerned readers. I'm sure that these books will prove beneficial not only to the researchers/students of ethnobotany but also to common interested people. These volumes will generate interest in their readers for carrying out studies on plant use culture that will lead to further documentation, sustainable use, and conservation of fascinating plant diversity of these mountain regions. 